\title{
Governance of conditional reimbursement practices in the
}

Netherlands

Wouter Boon $^{a^{*}}$ Luis Martins $^{b}$ and Marc Koopmanschap ${ }^{c}$

Please cite as: Boon, W.P.C., Martins, L., \& Koopmanschap, M. (2014) Governance of conditional reimbursement practices in the Netherlands. Health Policy 119(2), 180-185.

a Innovation Studies, Copernicus Institute of Sustainable Development, Utrecht University, Heidelberglaan 2, 3584 CS Utrecht, the Netherlands

${ }^{\mathrm{b}}$ Abbott, Hoofddorp, the Netherlands

${ }^{\text {c }}$ Institute of Health Policy and Management, Erasmus University, the Netherlands

* Corresponding author:

Wouter P.C. Boon

Innovation Studies, Copernicus Institute of Sustainable Development, Utrecht University, Heidelberglaan 2, 3584 CS Utrecht, the Netherlands

w.p.c.boon@uu.nl

Phone number: +31(0)302532708 


\title{
Governance of conditional reimbursement practices in the Netherlands
}

\begin{abstract}
When entering the market, orphan drugs are associated with substantial prices and a high degree of uncertainty regarding safety and effectiveness. This makes decision making about the reimbursement of these drugs a complex exercise. To advance on this, the Dutch government introduced a conditional reimbursement trajectory that requires a re-evaluation after four years. This article focuses on the origins, governance and outcomes of such a conditional reimbursement trajectory for orphan drugs. We find that the conditional reimbursement scheme is the result of years of discussion and returning public pressure about unequal access to expensive drugs. During the implementation of the scheme the actors involved went through a learning process about the regulation. Our analysis shows that previous collaborations or already existing organisational structures led to faster production of the required data on cost-effectiveness. However, cost-effectiveness evidence resulting from additional research seems to weigh less than political, judicial and ethical considerations in decision making on reimbursement of orphan drugs in the Netherlands.
\end{abstract}

\section{Keywords}

Drug reimbursement; Decision making; Conditional approvals; Orphan drugs; Pharmaceuticals 


\section{Introduction}

Orphan drugs target rare diseases, i.e. life-threatening or chronically debilitating illnesses with a low prevalence. Despite the small and often heterogeneous populations associated with these drugs, the number of orphan drugs entering the market has risen in recent years [1,2], amongst others because of supportive EU and US policies. Small patient pools mean that $\mathrm{R} \& \mathrm{D}$ costs need to be recouped from smaller sales, resulting in relative high prices [3]. Moreover, small and heterogeneous patient pools often indicate limited and weak clinical and economic data at time of product launch [4]. Such combination of substantial prices and high degrees of uncertainty leads to complex decision making about reimbursement of orphan drugs. Decisions on reimbursement of these drugs are taken against the background of two opposing perspectives: solidarity [5] versus efficient deployment of limited resources (e.g. [6]).

To deal with this complex decision making at time of market launch, the Dutch government introduced a conditional reimbursement trajectory that requires a re-evaluation after four years. This article concentrates on the origins, governance and outcomes of such a conditional reimbursement trajectory for orphan drugs. Although a wide range of scholars discussed the reimbursement of expensive orphan drugs $[3,4,6]$, this focus is interesting since little has been written about novel reimbursement routes. In addition, the societal relevance is reflected by the recurrent debates on innovation capacity of pharmaceutical companies [7] and on consequences for public health budgets and access to medicines [4]. More in general, reflecting on conditional reimbursement is in line with public policy interests to address uncertainties inherent to reimbursement decisions, e.g. in the form of managed entry agreements and risk-sharing schemes [8].

\section{Origins of conditional reimbursement}

Innovative orphan drugs, such as enzyme replacement therapies, answer to unmet medical needs associated with rare diseases. To benefit from centralising expertise, treatment with these specialised drugs is often initiated in university hospitals. However, centralisation led to concentration of costs in those hospitals, putting pressure on hospital budgets and to misalignment between priorities of hospital management and medical staff. In the end, inequalities in care provision between hospitals, labelled as 'healthcare postcode lottery', could ensue [9].

Problems with expensive specialist drugs entered the Dutch political arena for the first time in 1993 with the introduction of paclitaxel (which is not an orphan drug) and attracted attention of politics and media for over two decades. From 1995 onwards, patient organisations reported inequalities in prescriptions across hospitals and lobbied Parliament to address this issue. This led the ministry to support changes in medical guidelines, subsidies for paclitaxel prescriptions, and a registry to investigate the extent of the problem.

Only from 2001 onwards European-wide registered orphan drugs entered the Dutch market with the introduction of agalsidase alfa and alglucosidase beta for Fabry disease. Because of lacking data on cost-effectiveness and high unmet medical need, the ministry introduced a temporary, dedicated subsidy scheme. After another major political discussion, following problems with reimbursement of trastuzumab, an expensive (non-orphan) breast cancer drug, the minister introduced an instrument on financing expensive medicines that also explicitly included orphan drugs. 
Through this policy rule, hospitals obtained $100 \%$ compensation for their orphan drug costs and $80 \%$ for other expensive drugs. The policy rule applied to those drugs that were included on a positive list. Admission to the list was subject to criteria, including expected costeffectiveness and budget impact. Due to suboptimal data on cost-effectiveness at the moment of reimbursement decision making [10], inclusion was regarded as temporary, based on incomplete cost-effectiveness and therapeutic value data, and conditional to concrete research plans to enrich and complete the datasets. After four years a re-evaluation would happen, taking into account the produced data on budget impact, therapeutic value and costeffectiveness in daily practice. Such scheme could be regarded as an example of 'coverage with evidence development' [11].

In August 2012, four years after initiating conditional reimbursement of three orphan drugs for Pompe and Fabry disease (alglucosidase alfa, agalsidase alfa, alglucosidase beta), the first draft re-evaluation reports leaked to the press just before planned release by CVZ. These drafts reported that these drugs were too expensive (with annual treatment costs between 200,000-700,000 EUR) relative to the gain in quality of life and life expectancy. This reevaluation spurred a public outrage in the Netherlands in summer 2012 and reinvigorated public and political debate on (conditional) reimbursement policy as well as a reconsideration of the role of cost-effectiveness in reimbursement decision making.

\section{Evaluation of governance and outcomes of conditional reimbursement process}

When evaluating the first six years of the conditional reimbursement process (2006-2012; see Appendix for the methodology of the evaluation), we focused on two perspectives: the efficiency of the re-evaluation processes and the outcomes of these processes.

\subsection{Efficiency of governance of re-evaluation process}

In the re-evaluation process, the role of CVZ, the ministry and other governmental agencies was confined to defining procedures and assessment, leaving the coordination to a wide range of parties. Formally, the federation of hospitals is responsible for requesting inclusion of a drug on the conditional approval list and for producing additional data. The federation does not have expertise or direct incentives to be prime movers in the process, though. As a result of this clear lack of problem-ownership, in practice two coordination models emerged.

- In the first governance model (applying to orphan drugs labelled ' 1 ' in Figure 1) there is a clear expert centre in one university hospital that treats most patients in the Netherlands and performs research on the disease. The medical specialist-researchers already perform small-scale outcome studies, having good access to the patient population. Frequent and intimate interactions with patient organisations lead to effective research performance, e.g. in terms of patient recruitment and even cofounding of natural history registries.

- The second model articulates pharmaceutical companies as the major players (' 2 ' in

Figure 1). In most cases an acknowledged single expert centre is absent. The companies coordinate and subcontract the required studies in academic hospitals. In two orphan drug cases neither model applied (' 3 ' in Figure 1). The interactions between medical specialist-researcher, patient organisation and company were suboptimal or even lacking, and no actor was able to take the lead. 


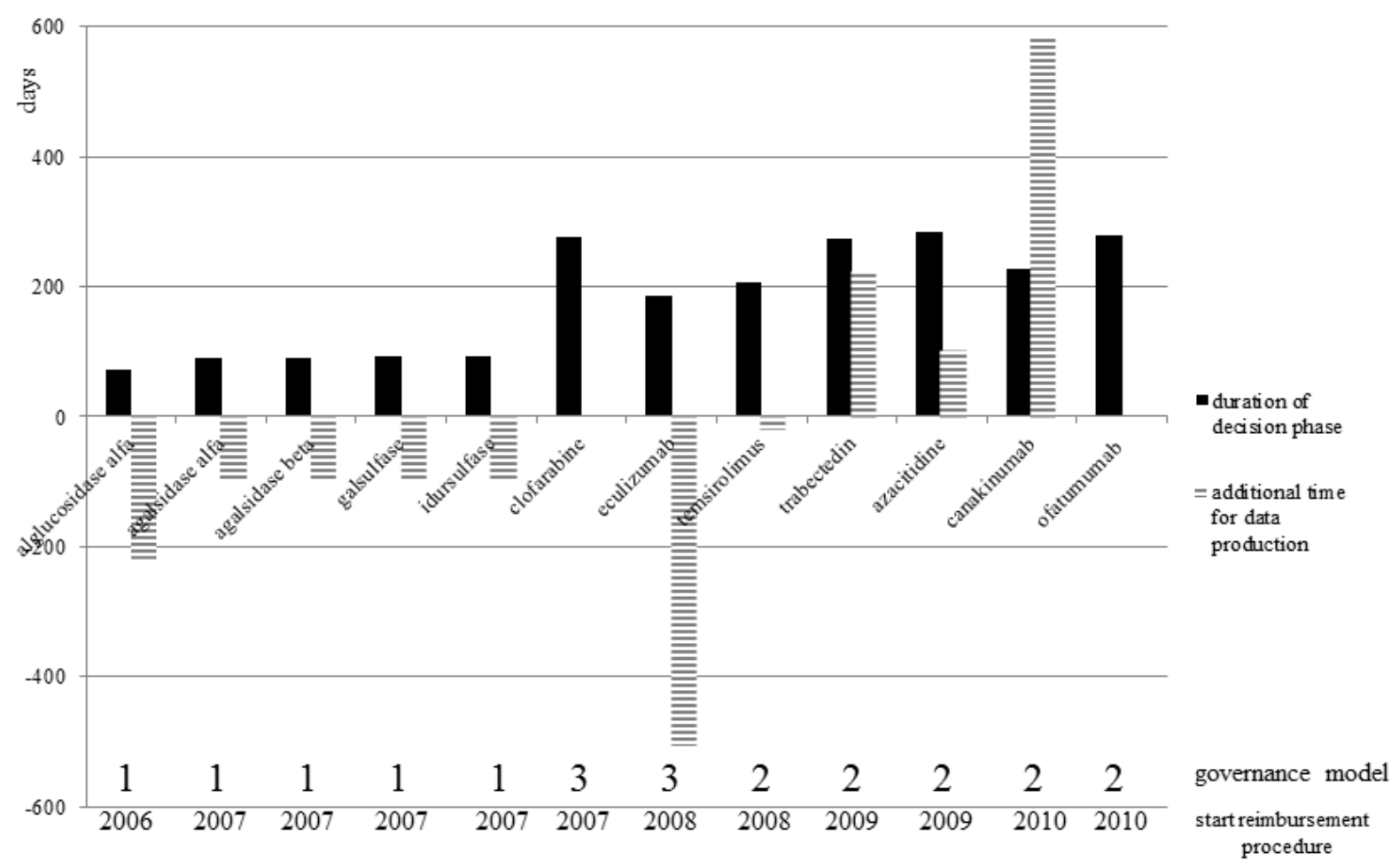

Figure 1: duration of decision phase and additional time for data production (see Appendix for methodology) in days. Numbers $1-2-3$ refer to the governance models. Negative additional time for data production signifies the number of days that the deadline of re-evaluation is before the end date of the HTA study.

Interview results favour the first governance model in terms of efficiency of conducting additional studies and re-evaluation, and respondents widely agree on the ineffectiveness of the third model. Figure 1 seems to support this. In this first period after the introduction of the policy rule, the decision-making process on the preparation of the cost-effectiveness studies and assessments by CVZ (black bars) of drugs associated with the medical-dominated governance model was shorter. The interview respondents suggest that particularly collaborations between medical specialist groups and patient organisations have a positive effect on the dynamics of designing cost-effectiveness studies.

However, the relationship between type of governance and the efficiency of the research process is far more complex due to various moderating variables. First, the type of disease may be influential. The medical-dominated model appears in cases of enzyme replacement therapies, which are mostly prescribed in one centre, whereas the company-dominated model applies to rare cancers that call for coordination between different centres in the Netherlands.

Efficiency of the models can also be the result of involved stakeholders becoming more knowledgeable about how to organise processes. Although the black bars in Figure 1 exhibit growing decision-making periods, there is another indicator that points to a learning effect. During the first period many starting problems and delays occurred, largely because of uncertainties about tasks and procedures, which first needed to be polished and fine-tuned. Key issue was setting up and conducting the cost-utility study. At first it was unclear which parameters needed to be included and how they would be measured. CVZ vigorously tried to define the evaluation criteria. Uncertainties about the process and criteria made it difficult for researchers and companies to adhere, especially since cost-effectiveness research had not 
been their 'core activity'. A specific complicating factor was that several processes needed to be started in parallel and ideally be interlinked. One of these parallel tracks was the possibility to obtain funding for the cost-effectiveness studies through a dedicated publicly-funded HTA program. Criteria used for evaluating proposals differed from criteria used by, for example, CVZ. The review and granting procedure led to delays in initiating the research. As a result, the HTA-studies would in some cases be finished - and thus producing data - several weeks after the deadline for re-evaluation dossier submission. This 'additional time for data production' is shown as grey bars in Figure 1; a negative time period signifying a shorter period in which data could be produced. For clofarabine and ofatumumab there were no applications for public support for cost-effectiveness studies. The extensive period of negative additional time related to eculizumab is the result of a series of rejections of the research proposal by the reviewers of the HTA program. The figure shows that the misalignment between procedures, and the associated 'additional time for data production' becomes positive for later drugs (trabectedin, azacitidine, canakinumab), indicating a possible learning effect and resulting in more time to produce and analyse data. In sum, the relation between the governance of cost-effectiveness studies and performance can also be mediated by learning effects.

\subsection{Outcomes of conditional reimbursement}

In addition to the process perspective, another way to evaluate the conditional reimbursement scheme is to look at the outcomes in terms of number of drugs approved, appeal procedures, reviews and revisions. To contextualise the Dutch results we made a comparison with France. A comparison between the Netherlands and France is warranted since both countries share common features, such as high GDP per capita and a perceived progressive approach towards orphan drug reimbursement within a common umbrella EU regulatory framework, albeit with distinct governance mechanisms (see e.g. [12]). There are also small differences in the number of reimbursed orphan drugs (Table 1). The reimbursement status of orphan drugs disagrees in 19\% of the cases (14 orphan drugs). Reasons include that reimbursement requests were pending (7) or reimbursement requests were not submitted (5). In 2 cases the French HTA agency (HAS) and the Dutch authorities did not agree. The average review period, as indicated by the interval between the date of marketing authorisation and medicine reimbursement, is shorter in France (193 days) than in the Netherlands (223 days).

Table 1: availability and reimbursement of orphan drugs - France vs. The Netherlands (see Appendix for methodology).

\begin{tabular}{|l|l|l|}
\hline Orphan designations & France & The Netherlands \\
\hline $\begin{array}{l}\text { Total authorised orphan } \\
\text { drugs in EU }\end{array}$ & 75 & 75 \\
\hline Launched by company & $68(91 \%)$ & $67(89 \%)$ \\
\hline Reimbursed & $61(81 \%)$ & $59(79 \%)$ \\
\hline In hospital & $41(55 \%)$ & $30(40 \%)$ \\
\hline Conditionally reimbursed & N/A & $14(19 \%)$ \\
\hline
\end{tabular}

Integral to conditional reimbursement is the re-evaluation of listed drugs. To investigate reevaluation, three indicators were used. First, in both France and the Netherlands any stakeholder can initiate on procedural grounds an appeal against a reimbursement decision. While there were records of stakeholders disagreeing with draft advices, e.g. regarding Pompe disease, and they even voiced their position during an official public meeting, formal appeals were not identified. 
Second, both countries have the possibility of revisions. In France reimbursement decisions are always temporary, i.e. there are systematic reimbursement reviews, whereas for the Netherlands the re-evaluation of listed orphan drugs is scheduled after four years $[13,14]$. Moreover, the French system has a quite liberal compassionate use programme, called Autorisation Temporaire d'Utilisation (ATU), which makes most orphan drugs available several months in advance of the release of the orphan drug into the market of each EU Member State.

Third, the extent to which actual revisions led to delisting or change of reimbursement status. A subsample was analysed consisting of 46 orphan designations with marketing authorisation obtained five years ago or before. In principle these designations are those most vulnerable to systematic (or ad hoc) reviews. In both countries, none of these drugs were delisted as a result of a reimbursement review. For the Netherlands this is surprising since cost-effectiveness evidence for this subset was in general quite poor. Actual use of cost-effectiveness analysis in dossiers is rare, observed only in $12 \%$ of the cases. For France, of the 46 designations, six ( $13 \%$ of total) had a drug assessment resulting in a downgrade. These downgrades did not result in delisting but the price and/or rate of reimbursement may have changed. For the 14 conditionally reimbursed orphan drugs in the Netherlands only 4 reassessments were finalised (with an average delay of 563 days), the rest are still pending (with some having a delay of 3 years and counting). CVZ gave a negative advice for 3 of the reassessed drugs, which showed costs per QALY above 200,000 EUR, but none of them were eventually delisted. However, the cost-effectiveness findings resulted into further research on narrowing down the criteria for effective use (stop and start criteria, precise patient subpopulations).

The lack of delistings seems to indicate that cost-effectiveness had at best a very modest impact on the reimbursement decisions of orphan drugs [15]. Discussions around the 3 reassessed drugs led to fierce public and political debate, as was already mentioned in section 2. Pressure from the general public, medical specialists and patient organisations weighted heavier than the negative advice based on cost-effectiveness data. Interview results reveal several reasons for this: the cost-effectiveness data were perceived as still rudimentary (which was to be expected after just four years of research); patient organisations had already anticipated on a possible negative advice by preparing elaborate media campaigns; after 4 years of (conditional) use, these drugs could be perceived as an acquired right; politicians and policymakers were not eager to define a threshold for the incremental cost-effectiveness ratio; many stakeholders regarding solidarity in healthcare as important, specifically for rare diseases no matter what the costs.

In sum, a comparison with France indicates that the Dutch conditional reimbursement scheme resulted in comparable and not necessarily faster access to orphan drugs. Also neither country had delistings. Cost-effectiveness evidence resulting from additional research seems to weigh less than political, judicial and ethical considerations.

\section{Conclusions and discussion}

Initial reimbursement decisions are always associated with a high level of uncertainty because data on effectiveness, safety and cost-effectiveness is seldom complete. This becomes even more prominent in the case of orphan drugs. A way of dealing with such uncertainty is by adopting the possibility to reimburse medicines on a conditional basis. This article focuses on the origins, governance and outcomes of such a conditional reimbursement trajectory for orphan drugs. Based on this, we can draw the following conclusions. 
First, during the implementation of the scheme the actors involved went through a learning process about the regulation. Our analysis shows that previous collaborations or already existing organisational structures, preferably including medical specialists and patient organisations, led to faster production of the required data on (cost)-effectiveness. Such mode of governance can easier overcome difficulties in transparency, legitimacy and feasibility of additional studies, which is in line with previous studies on conditional reimbursement [16]. Changing regulations after every public discussion, as the historical account in section 2 conveys, might therefore be problematic: actors in the field do not get enough time to adjust to new responsibilities and governance structures, leading to wasted learning effects.

Second, the conditional reimbursement scheme is the result of years of discussion and returning public controversy about unequal access to expensive drugs. The scheme was introduced to deal with this problem but the technical and administrative details still needed resolution. This fine tuning, and possibly also the lack of revisions, still masks the underlying debate about solidarity and limited resources in healthcare. Evaluation of the outcomes showed that the actual position/relevance of cost-effectiveness findings (in theory one of four decision criteria for advices on the basic benefit package in the Netherlands) is unclear and seems to be outweighed by political and ethical considerations that enter the debate after publication of the advice. This debate is populated by a wider range of stakeholders and quickly captures the attention of the general public. In response to these emotional public debates, CVZ decided to reconsider the relevance of cost-effectiveness as reimbursement criterion and initiated a consultation among physicians and the general public. Especially the general public was not convinced that cost-effectiveness should be important [17]. Acquiring more data on cost-effectiveness and postponement does not necessarily lead to less problematic and well-supported decision making. Reprieve may even create problems of its own: drugs are reimbursed based on low evidence for effectiveness, which causes society to pay a lot of money for an uncertain health effect. And by delaying final decision making, an additional argument of 'acquired right to care' enters the debate, making it difficult to delist medicines.

Third, also the quality of the cost-effectiveness findings was questioned by a wide range of actors, including the researchers. Four years might still be too short to produce good-quality data. The small number of patients, small-scale set-up and the ethical complexity of randomising patients to a placebo while the drug has already been approved, call for alternative study designs, including analysis of pooled international data (registries) of sufficient quality $[10,18]$. Nevertheless, the cases revealed that some cost-effectiveness thinking did enter the decision making: at least findings were discussed and even led to narrowing down the criteria for effective use, including, for example, clear stopping rules. The Netherlands might learn from the French system in which these criteria are established in a 'rolling review', by this avoiding too strict attachment to a four-year reassessment.

In principle, conditional financing is associated with a tentative and reflexive perspective on perceiving the value of expensive orphan drugs. This has potential advantages since it regulates the level of uncertainty during decision making and introduces the obligation to perform to the actors' best ability. As such, conditional reimbursement aligns well with the recent move to making the registration process of new medicines more flexible and tailormade, e.g. in terms of conditional approvals [19] and adaptive licensing [20]. These schemes require room for careful tinkering and learning in such a way that actors can carve out their responsibilities and processes. Moreover, delaying decision making and introducing elaborate reimbursement schemes still do not necessarily obviate political and ethical debate. 



\section{Appendix: methodology}

\section{Origins of conditional reimbursement}

The data for constructing the history of the conditional reimbursement scheme originates from the archives and public websites of the main actors (two cancer patient organisations, a medical specialist organisation, a federation representing pharmaceutical companies, the ministry of health, and Parliament). This data collection yielded 598 records, including minutes of board and committee meetings, letters, reports, evaluations, parliamentary proceedings, and annual reports. In addition, we conducted 15 interviews with representatives of these organisations ranging from chairmen and managing directors to researchers and secretaries. The interviews were aimed at clarifying archival information and uncovering the underlying assumptions behind the factual data.

\section{Governance of process}

The 12 orphan drugs that were listed under the orphan policy rule in the Netherlands were investigated in depth by conducting interviews with key informants in the period December 2010-February 2011. For 9 out of 12 we interviewed representatives of the company, patient organisation and medical specialist-researcher involved. We also interviewed representatives of companies that are responsible for 2 of the 3 orphan drugs that were still under consideration or were rejected. Moreover, representatives of the organisations that were involved in all cases, such as the health ministry, representatives of hospitals and the Health Care Insurance Board, were interviewed as well. Interviewees were contacted through e-mail. In most cases introduction was eased by the Dutch Steering Committee on Orphan drugs, which endorsed this research project. Interviews lasted around 60 minutes and consisted of two parts. In the first part the interviewee was taken through the history of the R\&D process, registration and reimbursement procedures. For this, we had prepared a timeline in which the key events were included, based on dossiers as produced by the registration and reimbursement authorities. In the second part of the interview the respondent was asked to reflect on the process and the conditional reimbursement policy in general. This was mostly an open-ended discussion but we prepared a list of topics that we wanted to treat. This list was based on 2 preparatory interviews with representatives of the Dutch Steering Committee on Orphan drugs. The list was continuously updated between interviews. The interviews were audiotaped and the outlines were subsequently transcribed and checked by the respondents. Analysis was done by finding patterns in the histories of the decision-making processes, as well as uncovering the evaluative aspects that were mentioned by the interview respondents. The latter was done by performing open coding.

Figure 1 is produced by compiling a database in which the start and stop dates of procedures is included. Data was obtained from the websites and annual reports of the organisations responsible for the procedures: Health Care Insurance Board CVZ (http://cvZ.nl/zorgpakket/cfhagenda; http://www.cvz.nl/binaries/live/CVZ_Internet/hst_content/nl/documenten/rubriek+zorgpa kket/cfh/overzicht+tijdelijk+opgenomen+intramurale+geneesmiddelen.pdf), the European Medicines Agency (http://www.ec.europa.eu/health/documents/communityregister/html/orphreg.htm), the Dutch Healthcare Authority, Dutch public health research funder ZonMw (http://www.zonmw.nl/nl/onderwerpen/alle-programmas/ farmacotherapie/projecten/dure-en-weesgeneesmiddelen; http://www.zonmw.nl/nl/onderwerpen/alle-programmas/ farmacotherapie/projecten/reviews-dure-geneesmiddelen/). 
12 out of 14 drugs included on the conditional reimbursement list are analysed; the excluded ones were either too new (no data available on re-evaluation procedure) or too old (one drug was 'inherited' from a previous policy measure and did not go through the full procedure).

Outcomes comparison between the Netherlands and France

In both countries we investigated the availability of orphan drugs. There are 75 medicines to be found in the European Union register (European Commission, 2013) that have market authorisation and an orphan drug designation. The period that is taken into account runs from 2000 (year in which the EU orphan drug directive became effective) until May 2013. Data on which orphan drugs have been launched in specific countries can be found in pharmacy databases (see below). In addition, we examined the reimbursement advice for a subsample of 20 designations and studied the occurrence of changes to the reimbursement status for a subsample of 46 designations in France and the Netherlands.

For the Netherlands we consulted the positive list of reimbursed drugs (http://wetten.overheid.nl/BWBR0018715/Bijlage4/geldigheidsdatum_02-06-2013), the drugs database of the Royal Dutch Association of Pharmacists

(http://farmanco.knmp.nl/tekortweesgeneesmiddel/) and the CVZ website (sections http://www.cvz.nl/, http://www.fk.cvz.nl/ and http://www.medicijnkosten.nl/).

For France we consulted the database of marketed drugs "Thesorimed" (http://theso.proddeux.thesorimed.org/), the website of the French Association of Health Insurance Funds (sections

http://www.codage.ext.cnamts.fr/codif/bdm_it/index.php?p_site; and http://www.codage.ext.cnamts.fr/codif/bdm_it/index_tele_ucd.php?p_site), the website of the French reimbursement authority (http://www.has-sante.fr/) and the database "Vidal" (http://www.vidal.fr/fiches-medicaments). In addition, to check the French database we conducted an interview with a drug reimbursement expert.

To investigate the reliability of the data, we crosschecked our list with the inventory of access and prices of orphan drugs across Europe done by Eurordis (see:

http://www.eurordis.org/content/access-orphan-drugs). The two lists agreed. 


\section{References}

[1] Heemstra HE, de Vrueh RLA, van Weely S, Büller HA, Leufkens HGM. Orphan drug development across Europe: bottlenecks and opportunities. Drug Discov Today 2008;13:670-6.

[2] Braun MM, Farag-El-Massah S, Xu K, Coté TR. Emergence of orphan drugs in the United States: a quantitative assessment of the first 25 years. Nat Rev Drug Discov 2010;9:519-22.

[3] Drummond MF, Wilson DA, Kanavos P, Ubel P, Rovira J. Assessing the economic challenges posed by orphan drugs. Int J Technol Assess Health Care 2007;23:36-42.

[4] Simoens S. Pricing and reimbursement of orphan drugs: the need for more transparency. Orphanet J Rare Dis 2011;6:42.

[5] Picavet E, Dooms M, Cassiman D, Simoens S. Orphan Drugs for Rare Diseases: Grounds for Special Status. Drug Dev Res 2012;73:115-9.

[6] McCabe C, Claxton K, Tsuchiya A. Orphan drugs and the NHS: should we value rarity? BMJ 2005;331:1016-9.

[7] Heemstra HE. From research on rare diseases to new orphan drug development (PhD thesis). Utrecht: Utrecht University; 2010.

[8] Sandmann FG, Franken MG, Steenhoek A, Koopmanschap MA. Do reassessments reduce the uncertainty of decision making? Reviewing reimbursement reports and economic evaluations of three expensive drugs over time. Health Policy 2013;112:28596.

[9] Gress S, Niebuhr D, Rothgang H, Wasem J. Criteria and procedures for determining benefit packages in health care. A comparative perspective. Health Policy 2005;73:7891.

[10] Kesselheim AS, Gagne JJ. Strategies for postmarketing surveillance of drugs for rare diseases. Clin Pharmacol Ther 2014;95:265-8.

[11] Hutton J, Trueman P, Henshall C. Coverage with evidence development: an examination of conceptual and policy issues. Int J Technol Assess Health Care 2007;23:425-32.

[12] Franken M, le Polain M, Cleemput I, Koopmanschap M. Similarities and differences between five European drug reimbursement systems. Int J Technol Assess Health Care 2012;28:349-57.

[13] Meyer F. Health Technology Assessment, pricing and reimbursement in France and European collaboration (presentation at University of Tokyo: http://www.pp.utokyo.ac.jp/HTA/events/2012-09-06/documents/hta20120906-meyer.pdf). Tokyo: 2012. 
[14] CVZ. Procedure herbeoordeling intramurale geneesmiddelen. Diemen: 2010.

[15] Franken M, Koopmanschap M, Steenhoek A. Health economic evaluations in reimbursement decision making in the Netherlands: Time to take it seriously? Z Evid Fortbild Qual Gesundhwes 2014.

[16] Niezen M, de Bont A, Stolk E, Eyck A, Niessen L, Stoevelaar H. Conditional reimbursement within the Dutch drug policy. Health Policy 2007;84:39-50.

[17] CVZ. Healthcare professionals on cost-effectiveness - proceeds of five kitchen table discussions [Zorgprofessionals over kosteneffectiviteit De opbrengst van vijf keukentafelgesprekken]. Diemen: 2013.

[18] Garattini S, Bertele V. Adjusting Europe’s drug regulation to public health needs. Lancet 2001;358:64-7.

[19] Boon WPC, Moors EHM, Meijer A, Schellekens H. Conditional approval and approval under exceptional circumstances as regulatory instruments for stimulating responsible drug innovation in Europe. Clin Pharmacol Ther 2010;88:848-53.

[20] Eichler H, Pignatti F, Flamion B, Leufkens HGM, Breckenridge A. Balancing early market access to new drugs with the need for benefit/risk data: a mounting dilemma. Nat Rev Drug Discov 2008;7:818-26. 